\title{
Outcomes of Phaco-viscocanalostomy in Primary Open Angle Glaucoma versus Pseudoexfoliation Glaucoma
}

\author{
Ebrahim Azaripour ${ }^{1}$, MD; Yaser Khakpour ${ }^{1}$, MD; Reza Soltani-Moghadam ${ }^{1}$, MD; Zahra Moravvej ${ }^{1}$, MD \\ Abdolreza Medghalchi ${ }^{1}$, MD; Hassan Behboudi ${ }^{1}$, MD; Yousef Alizadeh ${ }^{1}$, MD; Soheil Soltanipour ${ }^{2}$, MD \\ Shila Kianmehr', MS
}

${ }^{1}$ Eye Research Center, Department of Ophthalmology, Amiralmomenin Hospital, School of Medicine, Guilan University of Medical Sciences, Rasht, Iran

${ }^{2}$ GI Cancer Screening and Prevention Research Center, Department of Community Medicine, School of Medicine, Guilan University of Medical Sciences, Rasht, Iran

ORCID:

Ebrahim Azaripour: https://orcid.org/0000-0001-7309-1503

Reza Soltani-Moghadam: https://orcid.org/0000-0001-9661-3036

\section{Abstract}

Purpose: Viscocanalostomy represents an alternative to standard penetrating glaucoma surgery. The aim of this study is to compare the outcomes of combined phacoemulsification and viscocanalostomy in eyes with primary open-angle glaucoma (POAG) versus eyes with pseudoexfoliation glaucoma (PEXG).

Methods: In this prospective non-randomized comparative study, eyes with cataract and POAG or PEXG were enrolled. Pre- and postoperative data including best corrected visual acuity (BCVA), intraocular pressure (IOP), and the number of antiglaucoma medications administered were recorded at each visit. All patients underwent phacoviscocanalostomy. Complete success was defined as the IOP of $21 \mathrm{mmHg}$ or less without the administration of medication while a qualified success reported the same IOP parameters either with or without the administration of medication.

Results: Fifty-four eyes with POAG and fifty-four with PEXG underwent phacoviscocanalostomy. The mean follow-up time was $23.36 \pm 8.8$ months (range, 6-40 months). The mean postoperative IOP reduced significantly in both groups, although the mean IOP reduction was significantly greater in PEXG eyes (14.7 \pm 8.9 vs $10.1 \pm 7.7 \mathrm{mmHg})(P=0.05)$. At the final follow-up visit, the mean postoperative IOP was $14.1 \pm 2.1$ and $16.6 \pm 3.5 \mathrm{mmHg}$ in the PEXG and POAG eyes, respectively $(P=0.001)$. A complete success rate of $88.9 \%$ and $75.9 \%$ was achieved in PEXG and POAG eyes, respectively $(P=0.07)$. The qualified success rate was $100 \%$ in the PEXG and $85.2 \%$ in POAG groups $(P=0.03)$.

Conclusion: Phacoviscocanalostomy achieved significant IOP reduction and visual improvement in both POAG and PEXG patients. Our results indicated that in terms of IOP reduction, this procedure was more effective in treating PEXG.

Keywords: Primary Open-angle Glaucoma Intraocular Pressure; Phacoviscocanalostomy; Pseudoexfoliation

J Ophthalmic Vis Res 2021; 16 (4): 566-573 


\section{INTRODUCTION}

Primary open-angle glaucoma (POAG) and pseudoexfoliation glaucoma (PEXG) are chronic and progressive processes causing optic neuropathy. Pseudoexfoliation syndrome is featured by the deposition of specific fibrillar material in the anterior segment of the eye. ${ }^{[1,2]}$ This sedimentation of material on the trabecular meshwork (TM) may cause glaucoma and subsequent optic neuropathy. ${ }^{[3]}$

Various surgical procedures exist for managing glaucoma; these include penetrating and nonpenetrating filtering procedures and tube shunt surgery. ${ }^{[4,5]}$ Nonpenetrating procedures, such as deep sclerectomy and viscocanalostomy (VCS) have been designed to alleviate the complications of penetrating surgery ${ }^{[6]}$. VCS, as defined by Stegmann et al, involves the injection of high-viscosity sodium hyaluronate into the Schlemm's canal until aqueous outflow drainage is improved. ${ }^{[7]}$ It has been suggested that physiologic aqueous humor drainage may be attained without bleb formation.

Although trabeculectomy has proven to be more effective than nonpenetrating procedures in terms of IOP reduction, it is more prone to complications. ${ }^{[9]}$ VCS releases less inflammatory mediators causing lower rates of bleb failure. Phacoviscocanalostomy represents an alternative to standard phacotrabeculectomy with antimetabolites. ${ }^{[8]}$ Recent reports show good midterm results of phacoviscocanalostomy in eyes with medically uncontrolled glaucoma. ${ }^{[10-12]}$ In this study, we aim to compare the clinical outcomes of phacoviscocanalostomy with intraocular lens implantation in PEXG and POAG eyes.

\section{Correspondence to:}

Reza Soltani-Moghadam, MD. Eye Research Center, Eye Department, Amiralmomenin Hospital, School of Medicine, Guilan University of Medical Sciences, Rasht 4139637459, Iran

E-mail: reza_sm76@yahoo.com

Received: 02-12-2020 Accepted: 24-05-2021

Access this article online

Website: https://knepublishing.com/index.php/JOVR

DOI: $10.18502 /$ jovr.v16i4.9746

\section{METHODS}

This prospective comparative study was conducted on 108 of 108 eyes with PEXG (54 eyes) and POAG (54 eyes). The criteria used in choosing the participants for the study included eyes with medically uncontrolled POAG and PEXG (IOP > $21 \mathrm{mmHg}$ with maximum medical therapy and glaucomatous visual field defects) and visually significant cataract (visual acuity that significantly affects the patient's daily activities). Patients with other ocular pathologies and history of any ocular surgery or laser procedures were excluded from the study.

Demographic and preoperative data which included best corrected visual acuity (BCVA) in LogMAR, IOP (Goldmann tonometry), gonioscopic (four-mirror glass goniolens), and funduscopic findings were recorded. Standard automated perimetry (Humphrey Field Analyzer, program 242) and glaucoma severity staging done according to Hodapp-Parrish-Anderson criteria was also obtained from all patients. Postoperative followups were conducted initially on day seven and subsequently at $1,3,6,12,24,30$, and 40-month intervals. Complete slit-lamp examinations were performed in each follow-up visit along with BCVA and IOP measurements.

The study was conducted in accordance with the principles of the Declaration of Helsinki and was approved by the local Ethics Committee. Patients were informed about the aim of the study and written informed consent was obtained from all participants before the surgery. At the ophthalmology clinic, all eyes underwent surgery and follow-up examinations, both of which were performed by a single surgeon (EA).

Statistical analysis was calculated using the SPSS V19.1 application. Preoperative and postoperative data were compared using paired sample $t$-test and Wilcoxon sign test (for nonparametric data). Comparison between the two

This is an open access journal, and articles are distributed under the terms of the Creative Commons Attribution-NonCommercial-ShareAlike 4.0 License, which allows others to remix, tweak, and build upon the work non-commercially, as long as appropriate credit is given and the new creations are licensed under the identical terms.

How to cite this article: Azaripour E, Khakpour $Y$, Soltani-Moghadam R, Moravvej Z, Medghalchi A, Behboudi H, Alizadeh Y, Soltanipour S, Kianmehr S. Outcomes of Phaco-viscocanalostomy in Primary Open Angle Glaucoma versus Pseudoexfoliation Glaucoma. J Ophthalmic Vis Res 2021;16:566573 
groups was performed using student's $t$-test and the Mann-Whitney $U$ test (for nonparametric data). Comparisons of the outcome of the glaucoma at different stages were done using the KruskalWallis test. The frequency of intraoperative and postoperative complications was assessed in both groups. $P$-value $<0.05$ was considered to be statistically significant. According to international consensus statements, complete success was characterized as an IOP $\leq 21 \mathrm{mmHg}$ without antiglaucoma medication while qualified success was determined as achieving the same IOP values but either with or without antiglaucoma medication. Failure was described as an IOP $>21 \mathrm{mmHg}$ with the administration of medication, or when an eye required further glaucoma drainage surgery. ${ }^{[13]}$

\section{Surgical Technique}

Surgical procedures were performed either utilizing peribulbar or general anesthesia depending on the patient's medical history. Prior to performing VCS, patients underwent standard phacoemulsification with intraocular lens implantation. Phacoemulsification was performed through a temporal $2.8 \mathrm{~mm}$ clear cornea incision. After executing continuous circular capsulorhexis (CCC), the nucleus was emulsified using "stop and chop" or "divide and conquer" techniques. After complete nuclear and cortical material removal, a foldable intraocular lens was inserted into the capsular bag. In order to perform VCS, a fornixbased conjunctival peritomy was made and a square-shaped superficial scleral flap $(5.0 \times 5.0$ $\mathrm{mm}$ ) was created by a crescent blade. A second deeper scleral flap $(4.0 \times 4.0 \mathrm{~mm})$ was dissected and continued anteriorly to the scleral spur to expose the trabeculo-descemet membrane (TDM). The deep scleral flap and the roof of Schlemm's canal were cut to facilitate aqueous humor drainage. Sodium hyaluronate 1.4\% (Healon GV) was then injected into the Schlemm's canal. The superficial scleral flap was closed tightly with two interrupted 10-0 nylon sutures, and the conjunctival wound was sutured with 10-0 nylon.

\section{RESULTS}

A total of 108 eyes of 108 patients with PEXG (54 eyes) and POAG (54 eyes) were included in the study. The mean age of the participants was 70.3 \pm 8.03 years and 61 (56.5\%) of them were male. All patients completed postoperative follow-up for at least 12 months. The mean follow-up time after surgery was $23.36 \pm 8.8$ months (range 12-40 months). The demographic and baseline data of the two groups is shown in Table 1. There was no significant difference between the PEXG and the POAG groups in terms of glaucoma severity $(P=$ 0.585). The gonioscopy results revealed slightly narrower angles in the PEXG group as compared to the POAG group, although this difference was not statistically significant $(79.6 \%$ in PEXG vs $100 \%$ in POAG were classified as open-angle, $P=0.057$ ).

All patients had a significant improvement in BCVA, postoperatively $(P=0.001)$. There was no statistically significant difference in terms of postoperative BCVA between the POAG and the PEXG groups $(P=0.88)$.

The mean IOP decreased significantly one week after surgery in both groups $(P=0.001)$ and remained significantly lower than its preoperative value at all follow-up visits [Figure 1]. The mean IOP was significantly lower in the PEXG group as compared to the POAG group at the 12- and 24month follow-up visits. This trend continued until the last follow-up visit although not statistically significant [Table 2]. At the final follow-up, the mean postoperative IOP reduction in the PEXG group was significantly greater than the POAG group (14.7 \pm 8.9 vs $10.1 \pm 7.7 \mathrm{mmHg}, P=0.01)$.

Overall, the number of antiglaucoma medications required reduced significantly after surgery $(1.2 \pm 1.2$ to $0.12 \pm 0.46, P=0.001)$, although not statistically different between the two study groups [Table 1].

The most common intraoperative and postoperative complication of phacoviscocanalostomy was microperforation of the TDM and fibrin formation, respectively [Table 3]. At the final follow-up, complete success was noted in $88.9 \%$ (48 eyes) and $75.9 \%$ (41 eyes) of the PEXG and POAG patients, respectively $(P=0.07)$. Qualified success was achieved in all eyes (100\%) of the PEXG group and $85.2 \%$ of the POAG group $(P=0.03)$. In both the PEXG and POAG groups, complete success rates were not significantly different among the varying stages of glaucoma $(P=0.587$ for stages of PEXG and $P=$ 0.252 for stages of POAG).

The cumulative probability estimated using the Kaplan-Meier survival curve of complete success 
Table 1. Demographic data, baseline, and postoperative data at final follow-up visit in the study groups

\begin{tabular}{|c|c|c|c|}
\hline & PEXG (n=54) & POAG $(n=54)$ & P-value \\
\hline Age (yr) & $72.0 \pm 7.8$ & $68.5 \pm 7.9$ & 0.02 \\
\hline Gender (male/female) & $33 / 21$ & $28 / 26$ & 0.33 \\
\hline Preoperative BCVA (logMAR) & $0.97 \pm 0.4$ & $0.91 \pm 0.4$ & $0.32^{*}$ \\
\hline Postoperative BCVA (LogMAR) & $0.38 \pm 0.35$ & $0.39 \pm 0.35$ & $0.88^{*}$ \\
\hline Preoperative IOP $(\mathrm{mmHg})$ & $28.5 \pm 9.0$ & $24.0 \pm 8.7$ & $0.004^{*}$ \\
\hline Preoperative antiglaucoma medication (n) & $1.06 \pm 1.02$ & $1.5 \pm 1.32$ & 0.07 \\
\hline Postoperative antiglaucoma medication (n) & $0.11 \pm 0.31$ & $0.28 \pm 0.57$ & 0.06 \\
\hline Follow-up time (months) & $23.7 \pm 8.2$ & $22.9 \pm 9.4$ & 0.67 \\
\hline Cup-to-disc ratio & $0.84 \pm 0.1$ & $0.85 \pm 0.1$ & 0.85 \\
\hline Severity, n (\%) & & & $0.585^{\dagger}$ \\
\hline Mild & $4(7.4)$ & $6(11.1)$ & \\
\hline Moderate & 15 (27.8) & $11(20.4)$ & \\
\hline Severe & $35(64.8)$ & 37 (68.5) & \\
\hline
\end{tabular}

Data is expressed as Mean $\pm \mathrm{SD}$

POAG, primary open-angle glaucoma; PEXG, pseudoexfoliation glaucoma; BCVA, best-corrected visual acuity; IOP, intraocular pressure; SD, standard deviation

*Mann-Whitney $U$ test; ${ }^{\dagger}$ Chi-square test

Table 2. Mean IOP ( $\mathrm{mmHg}$ ) over 40 months (ms) follow-up in the two study groups

\begin{tabular}{lcccccccc}
\hline & $\begin{array}{c}1 \text { week } n \\
=108\end{array}$ & $1 \mathrm{~m} n=108$ & $3 \mathrm{~ms} n=108$ & $6 \mathrm{~ms} n=108$ & $12 \mathrm{~ms} n=108$ & $24 \mathrm{~ms} n=92$ & $30 \mathrm{~ms} n=84$ & $40 \mathrm{~ms} n=76$ \\
\hline PEXG & $10.5 \pm 3.9$ & $13.0 \pm 4.3$ & $13.7 \pm 2.7$ & $16.4 \pm 2.3$ & $14.1 \pm 2.3$ & $14.5 \pm 2.1$ & $15.2 \pm 2.1$ & $13.5 \pm 1.9$ \\
POAG & $9.9 \pm 3.1$ & $12.9 \pm 3.0$ & $13.2 \pm 2.5$ & $15.4 \pm 4.6$ & $15.5 \pm 3.0$ & $16.8 \pm 3.7$ & $16.3 \pm 2.8$ & $15.8 \pm 1.3$ \\
P-value* & 0.35 & 0.33 & 0.45 & 0.62 & 0.009 & 0.001 & 0.17 & 0.08
\end{tabular}

Data is expressed as Mean \pm SD

POAG, primary open-angle glaucoma; PEXG, pseudoexfoliation glaucoma

${ }^{*}$ Students $t$-test

(IOP $<21 \mathrm{mmHg}$ without medication) in the study groups is illustrated in Figure 2. A moderately stable level of surgical success was achieved as was noted during the follow-up periods. No patients required additional glaucoma surgery.

\section{DISCUSSION}

Viscocanalostomy in comparison to trabeculectomy, is a nonpenetrating technique known to decrease intra- and postoperative complications of glaucoma surgery. ${ }^{[14-16]}$ Owing to its pathophysiology, PEXG eyes are more prone to trabeculectomy-related complications. ${ }^{[17,18]}$ The blood-ocular barrier dysfunction and iris stromal vasculopathy may contribute to postoperative inflammation, fibrin formation, and IOP elevation. ${ }^{\text {[19] }}$

Stegmann et al in 1999 reported the first study of VCS on 214 eyes with open-angle glaucoma (OAG) with a mean follow-up of 35 months. ${ }^{[7]}$ They achieved a complete success rate (IOP $\leq 22 \mathrm{mmHg}$ without medication) and a qualified success rate (IOP $\leq 22 \mathrm{mmHg}$ with medication) of $82.7 \%$ and $89 \%$, respectively. Another study in 2004 reported a complete success rate of $35.3 \%$ and a qualified success rate of $73.9 \%$ after three years, following the VCS in POAG eyes. ${ }^{[20]}$ Performing VCS without phacoemulsification, in addition to the reported higher preoperative IOP $(36.0 \pm 8.0 \mathrm{mmHg})$ as compared to our study $(24.0 \pm 8.7 \mathrm{mmHg})$ may 
Table 3. Intraoperative and postoperative complications

Complications

PEXG n (\%)

POAG n (\%)

Descemet membrane microperforation

$3(5.6)$

2 (3.7)

Fibrin reaction

$5(9.2)$

3 (5.6)

Zonular dehiscence

3 (5.6)

0

POAG, primary open-angle glaucoma; PEXG, pseudoexfoliation glaucoma

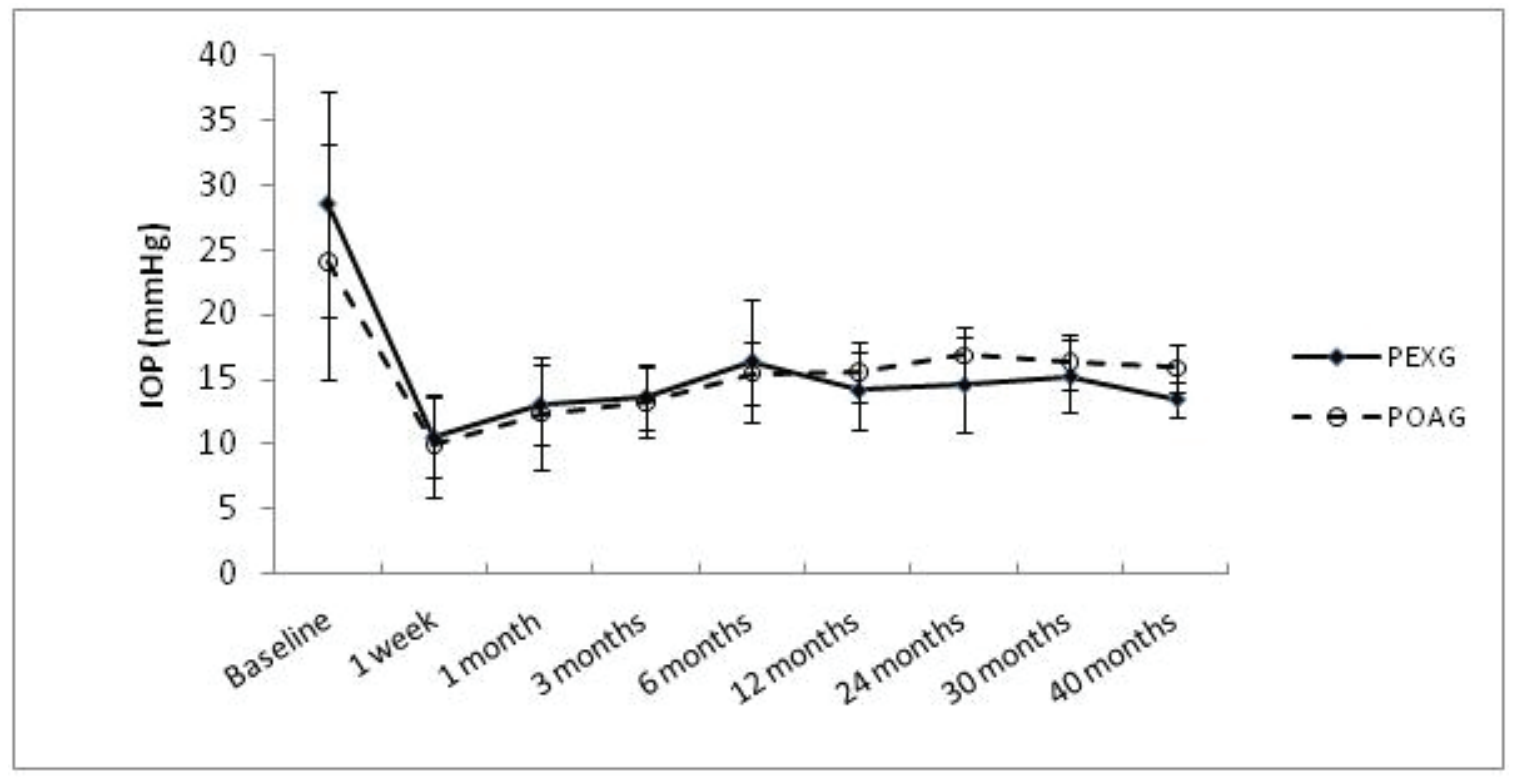

Figure 1. Mean intraocular pressure (IOP) over time in two groups. PEXG, pseudoexfoliation glaucoma; POAG, primary open angle glaucoma.

be reasons for the lower success rates achieved in their study. The combined procedures of phacoemulsification and nonpenetrating glaucoma surgery is being used frequently as it has produced good evidence of visual improvements and longterm IOP control. ${ }^{[16,21]}$ A 12-year follow-up study by Gunenc et al showed superior success rates in eyes undergoing phacoviscocanalostomy as compared with performing VCS on its own. ${ }^{[22]}$

In our present study, we noted significant reduction in the mean IOP levels after performing phacoviscocanalostomy in both POAG and PEXG eyes. However, significantly lower IOP levels were achieved in PEXG eyes at the 12- and 24-month follow-up visits. This finding may be partly due to significantly higher preoperative IOP in the PEXG patients. At all postoperative follow-ups, a similar comparative study reported significantly lower mean IOP in the PEXG group after performing phacoviscocanalostomy. ${ }^{[23]}$
In a retrospective one-year study, Moghimi et al reported a $37 \%$ complete success rate (defined as $I O P \leq 21 \mathrm{mmHg}$ without medication) in 46 OAG eyes (including PEXG) having undergone phacoviscocanalostomy. ${ }^{[24]}$ In another study, eyes with advanced glaucoma having undergone phacoviscocanalostomy achieved complete and qualified success rates of $30.6 \%$ and $80 \%$, respectively. ${ }^{[25]}$ Stangos et al performed phacoviscocanalostomy on 50 eyes with medically uncontrolled OAG and clinically significant agerelated cataract. The results reported an overall success rate (IOP $\leq 20 \mathrm{mmHg}$ with or without medication) of $82 \%$ and a complete success rate (IOP $\leq 20 \mathrm{~mm} \mathrm{Hg}$ without medication) of $67 \%$ at the 36-month follow-up visit. ${ }^{[26]}$ In comparison to the aforementioned studies, ${ }^{[24-26]}$ we experienced higher rates of complete and qualified success. Our study also indicated higher rates of complete success in the PEXG as compared to that of the POAG eyes (88.9 vs $75.9 \%$ ). All PEXG eyes in 


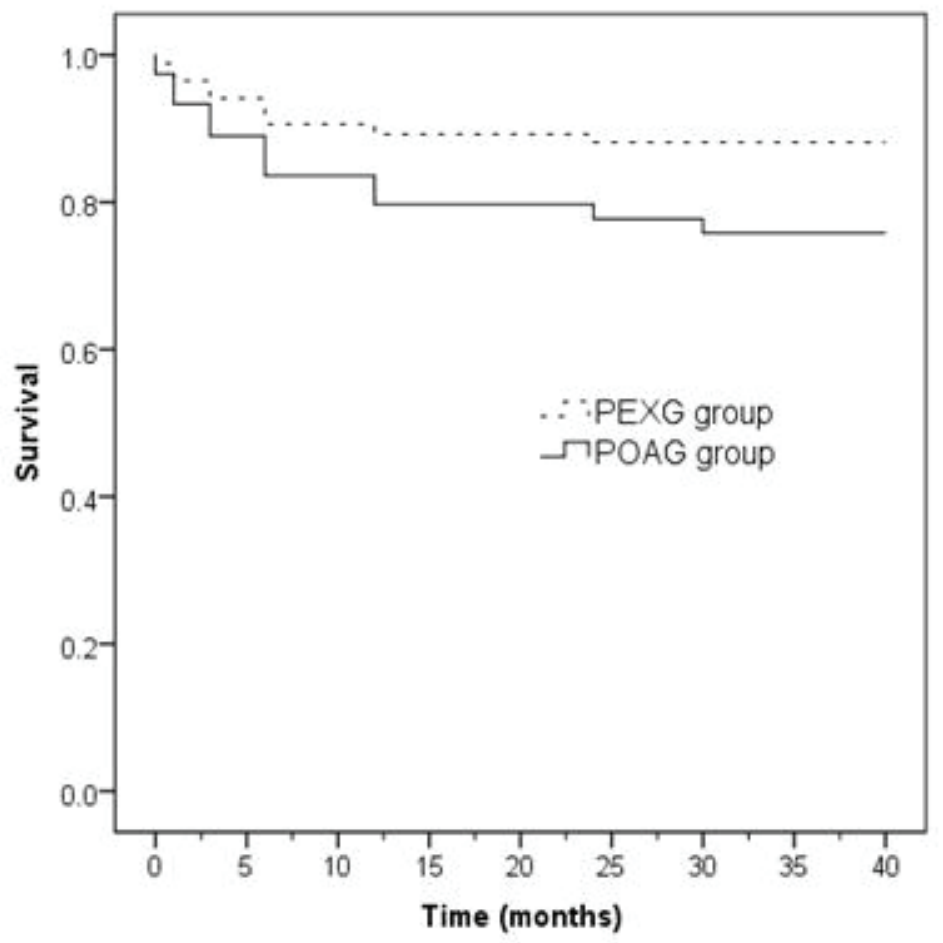

Figure 2. Kaplan-Meier survival curve of complete success (IOP $<21 \mathrm{mmHg}$ without medication) in pseudoexfoliation glaucoma (PEXG) and primary open-angle glaucoma (POAG).

our study and $85.2 \%$ of the POAG eyes achieved qualified success. Likewise, Awadalla et al reported complete surgical success of $93.3 \%$ in PEXG eyes and of $83.3 \%$ in POAG eyes. ${ }^{[23]}$ Their study resulted in qualified success of all patients in both groups. Wishart et al also achieved higher complete success rates (defined as IOP $\leq 18 \mathrm{mmHg}$ without medication) in PEXG as compared to POAG eyes after a mean follow-up of six years $(95 \%$ and $76 \%$, respectively), they also experienced $100 \%$ qualified success in PEXG and $90.2 \%$ in POAG eyes. ${ }^{[27]}$ According to our current study and the studies mentioned above, phacoviscocanalostomy success rates were higher in PEXG as compared to POAG patients. ${ }^{[13,14,23]}$

The type of glaucoma may also influence IOP reduction after cataract surgery. Numerous studies over the past few decades have shown that cataract surgery leads to a sustained decrease in IOP in POAG and pseudoexfoliation patients. ${ }^{[28]}$ Masis et al performed a systematic review and meta-analysis of the clinical data to estimate the net effect of cataract surgery on IOP. A total of 37 treatment arms from 32 different studies from January 1997 to January 2017 were included. For angle-closure glaucoma, results showed an IOP decrease of $-6.4 \mathrm{mmHg}(95 \% \mathrm{Cl}$ : -9.4 to -3.4$)$ at final follow-up (12 months and longer). For the OAG group, there was an overall IOP change of -2.7 $\mathrm{mmHg}(95 \% \mathrm{Cl}:-3.7$ to -1.7$)$ from the baseline and for PEXG there was an overall IOP change of -4.8 $\mathrm{mmHg}(95 \% \mathrm{Cl}:-7.5$ to -2.0$) .{ }^{[29]}$

In most studies, patients with PEXG have a significantly greater drop in IOP after phacoemulsification than patients with POAG. Reduction of IOP has been previously reported in patients with PEXG after phacoemulsification was performed. It is suggested that phacoemulsification may remove the fibrillar material source in PEX eyes and facilitate aqueous humor drainage in the trabecular meshwork. ${ }^{[25]}$ Moreover, wider angles resulting from phacoemulsification and cataract removal increases the aqueous outflow from the TM and Schlemm canal. ${ }^{[30]}$ Therefore, in patients with significant cataract and uncontrolled glaucoma, performing phacoviscocanalostomy can result in better outcomes than performing VCS alone.

Comparable to previous reports, microperforation of the TDM was the most common 
intraoperative complication while fibrin formation was the most common postoperative complication of phacoviscocanalostomy. ${ }^{[14,23]}$

As compared to prior studies, the main advantage of our study in comparing phacoviscocanalostomy in PEXG and POAG was the use of a relatively higher number of cases with longer follow-up periods. According to literature, longer follow-up periods for patients tends to show a decrease in the success rate. ${ }^{[12,22]}$ We, however, demonstrated rather stable success rates after the 12 month follow-up session until the last follow-up visit at 40 months post operation.

Our study had several limitations. Firstly, IOP monitoring and grouping were done by an examiner who was not masked to the groups which might contribute to possible bias in the results. Considering the average follow-up time of this study, we recommend longer-term followup sessions for glaucoma patients undergoing phacoviscocanalostomy. It is also recommended that for better comparison of VCS in PEXG and POAG, the procedure should be performed in patients who have not had cataract removal.

Multiple factors may affect the reported success rate of phacoviscocanalostomy, including: preoperative IOP levels, different levels of the surgeons' experience, the surgical techniques chosen, the follow-up time, the various criteria used to determine the success rate and the patients' medical history. Longer follow-up durations could affect the success rate, mainly due to the time taken for the deposition of fibrillar material in the angle of pseudoexfoliative eyes and the scarring ostia of the Schlemm's canal.

Various definitions of the success rate, study designs, and the execution of multiple followup times are contributory factors toward making comparison between studies challenging.

For future studies, it is recommended that authors continue to utilize the international criteria for intraocular pressure (IOP) levels, to ensure that the comparison between studies remain practical.

In summary, phacoviscocanalostomy is a safe and effective procedure in achieving IOP reduction for both PEXG and POAG eyes, as it increases BCVA and decreases trabeculectomyrelated complications. Therefore, this technique is recommended for eyes with therapeutically uncontrolled PEXG and POAG with cataract.

\section{Financial Support and Sponsorship}

None.

\section{Conflicts of Interest}

All authors declare that they have no conflicts of interests.

\section{REFERENCES}

1. Naumann GO, Schlötzer-Schrehardt $U$, Küchle $M$. Pseudoexfoliation syndrome for the comprehensive ophthalmologist: intraocular and systemic manifestations. Ophthalmology 1998;105:951-968.

2. Rao KN, Ritch R, Dorairaj SK, Kaur I, Liebmann JM, Thomas $\mathrm{R}$, et al. Exfoliation syndrome and exfoliation glaucomaassociated LOXL1 variations are not involved in pigment dispersion syndrome and pigmentary glaucoma. Mol Vis 2008;14:1254.

3. Leske MC, Heijl A, Hussein M, Bengtsson B, Hyman L, Komaroff E. Factors for glaucoma progression and the effect of treatment: the early manifest glaucoma trial. Arch Ophthalmol 2003;121:48-56.

4. Caretti L, Buratto L. Non-penetrating glaucoma surgery (NPGS): viscocanalostomy, deep sclerectomy and canaloplasty. Glaucoma Surgery: Springer; 2018. p. 23-40.

5. Chiselita D. Non-penetrating deep sclerectomy versus trabeculectomy in primary open-angle glaucoma surgery. Eye 2001;15:197-201.

6. Sayed MS, Lee RK. Recent advances in the surgical management of glaucoma in exfoliation syndrome. $J$ Glaucoma 2018;27:S95.

7. Stegmann R, Pienaar A, Miller D. Viscocanalostomy for open-angle glaucoma in black African patients. J Cataract Refract Surg 1999;25:316-322.

8. O'Brart D, Shiew M, Edmunds B. A randomised, prospective study comparing trabeculectomy with viscocanalostomy with adjunctive antimetabolite usage for the management of open angle glaucoma uncontrolled by medical therapy. Br J Ophthalmol 2004;88:1012-1017.

9. Gabai A, Cimarosti R, Battistella C, Isola M, Lanzetta P. Efficacy and safety of trabeculectomy versus nonpenetrating surgeries in open-angle glaucoma: a meta-analysis. J Glaucoma 2019;28:823-833.

10. Ho DK-h, Garrick A, Aazem S, Mathews D. Effect of primary phacoviscocanalostomy/viscocanalostomy on intraocular pressure of normal tension glaucoma patients: 3-year results. BMC Ophthalmol 2017;17:1-6.

11. Mandić Z, Sarić D, Bojić L. Visco and phacoviscocanalostomy in managing glaucoma patients. Coll Antropol 2002;26:165-169.

12. Shoji T, Tanito M, Takahashi H, Park M, Hayashi K, Sakurai $Y$, et al. Phacoviscocanalostomy versus cataract surgery only in patients with coexisting normal-tension glaucoma: midterm outcomes. J Cataract Refract Surg 2007;33:1209-1216. 
13. Hassan K, Awadalla $M$. Results of combined phacoemulsification and viscocanalostomy in patients with cataract and pseudoexfoliative glaucoma. Eur J Ophthalmol 2008;18:212-219.

14. Park M, Hayashi K, Takahashi H, Tanito M, Chihara E. Phaco-viscocanalostomy versus phaco-trabeculotomy: a middle-term study. J Glaucoma 2006;15:456-461.

15. Tanito M, Park M, Nishikawa M, Ohira A, Chihara E. Comparison of surgical outcomes of combined viscocanalostomy and cataract surgery with combined trabeculotomy and cataract surgery. Am J Ophthalmol 2002;134:513-520.

16. Mendrinos E, Mermoud A, Shaarawy T. Nonpenetrating glaucoma surgery. Surv Ophthalmol 2008;53:592-630.

17. Ritch R, Schlötzer-Schrehardt U. Exfoliation syndrome. Surv Ophthalmol 2001;45:265-315.

18. Conway RM, Schlötzer-Schrehardt $U$, Küchle $M$, Naumann GO. Pseudoexfoliation syndrome: pathological manifestations of relevance to intraocular surgery. Clin Experiment Ophthalmol 2004;32:199-210.

19. Cursiefen C, Hammer T, Küchle M, Naumann GO, Schlötzer-Schrehardt U. Pseudoexfoliation syndrome in eyes with ischemic central retinal vein occlusion: a histopathologic and electron microscopic study. Acta Ophthalmol Scand 2001;79:476-478.

20. Yalvac IS, Sahin M, Eksioglu U, Midillioglu IK, Aslan BS, Duman S. Primary viscocanalostomy versus trabeculectomy for primary open-angle glaucoma: threeyear prospective randomized clinical trial. J Cataract Refract Surg 2004;30:2050-2057.

21. Want A, Ho DK-H, Karri B, Mathews D. The efficacy of viscocanalostomies and combined phacoemulsification with viscocanalostomies in the treatment of patients with glaucoma: a non-randomised observational study. BMC Ophthalmol 2018;18:1-8.
22. Gunenc U, Ozturk T, Arikan G, Kocak N. Long-term results of viscocanalostomy and phacoviscocanalostomy: a twelve-year follow-up study. Int J Ophthalmol 2015;8:1162.

23. Awadalla MA, Hassan KM. Phacoviscocanalostomy in pseudoexfoliation glaucoma versus primary open-angle glaucoma. Can J Ophthalmol 2011;46:77-82.

24. Moghimi S, Hamzeh $\mathrm{N}$, Mohammadi $\mathrm{M}$, Khatibi $\mathrm{N}$, Bowd C, Weinreb RN. Combined glaucoma and cataract surgery: comparison of viscocanalostomy, endocyclophotocoagulation, and ab interno trabeculectomy. J Cataract Refract Surg 2018;44:557565.

25. Tsagkataki M, Bampouras T, Choudhary A. Outcomes of viscocanalostomy and phaco-viscocanalostomy in patients with advanced glaucoma. Graefes Arch Clin Exp Ophthalmol 2018;256:1481-1487.

26. Stangos AN, Mavropoulos A, Sunaric-Megevand G. Phacoviscocanalostomy for open-angle glaucoma with concomitant age-related cataract. Clin Ophthalmol 2007;1:497.

27. Wishart PK, Wishart MS, Choudhary A, Grierson I. Long-term results of viscocanalostomy in pseudoexfoliative and primary open angle glaucoma. Clin Experiment Ophthalmol 2008;36:148-155.

28. Friedman DS, Jampel HD, Lubomski LH, Kempen JH, Quigley $\mathrm{H}$, Congdon $\mathrm{N}$, et al. Surgical strategies for coexisting glaucoma and cataract: an evidence-based update. Ophthalmology 2002;109:1902-1913.

29. Masis M, Mineault PJ, Phan E, Lin SC. The role of phacoemulsification in glaucoma therapy: a systematic review and meta-analysis. Survey of ophthalmology 2018;63:700-710.

30. Park M, Tanito M, Nishikawa M, Chihara E. Ultrasound biomicroscopy of intrascleral lake after viscocanalostomy and cataract surgery. J Glaucoma 2004;13:472-478. 